\title{
International survey of contact lens fitting for myopia control in children
}

DOI:

10.1016/j.clae.2019.06.008

\section{Document Version}

Accepted author manuscript

Link to publication record in Manchester Research Explorer

\section{Citation for published version (APA):}

The International Contact Lens Prescribing Survey Consortium (2019). International survey of contact lens fitting for myopia control in children. Contact Lens and Anterior Eye. https://doi.org/10.1016/j.clae.2019.06.008

\section{Published in:}

Contact Lens and Anterior Eye

\section{Citing this paper}

Please note that where the full-text provided on Manchester Research Explorer is the Author Accepted Manuscript or Proof version this may differ from the final Published version. If citing, it is advised that you check and use the publisher's definitive version.

\section{General rights}

Copyright and moral rights for the publications made accessible in the Research Explorer are retained by the authors and/or other copyright owners and it is a condition of accessing publications that users recognise and abide by the legal requirements associated with these rights.

\section{Takedown policy}

If you believe that this document breaches copyright please refer to the University of Manchester's Takedown Procedures [http://man.ac.uk/04Y6Bo] or contact uml.scholarlycommunications@manchester.ac.uk providing relevant details, so we can investigate your claim.

\section{OPEN ACCESS}




\title{
Manuscript Details
}

\section{Manuscript number}

Title
CLAE_2019_16_R1

International survey of contact lens fitting for myopia control in children

\section{Article type}

Full Length Article

\begin{abstract}
Purpose. To determine the extent of contact lens fitting for myopia control (MC) in children (defined as $\leq 17$ years of age) worldwide and to characterize the associated demographics and fitting patterns. Methods. Survey forms were sent to contact lens fitters in 66 countries between January and March every year for eight consecutive years (2011 to 2018, inclusive). Practitioners were asked to record data relating to the first 10 contact lens fits performed after receiving the survey form. Data were analysed for those countries reporting $\geq 100$ contact lens fits to children. Results. Data were analysed for $535 \mathrm{MC}$ fits and 23,830 other (non-MC) lens fits undertaken in 31 countries reporting $\geq 100$ contact lens fits to children, with $52.1 \%$ of $M C$ fits and $12.0 \%$ of non-MC fits being with rigid lenses $(p<0.0001)$. Overall, MC lenses represented $2.3 \%$ of all contact lens fits to children, with significant differences between nations ( $p$ $<0.0001$ ), ranging from no MC fits recorded in the Czech Republic, Greece, Japan, South Korea and Puerto Rico, to $24.9 \%$ in Austria. There has been an increase in contact lens fitting for MC over the survey period $(p<0.0001)$. MC contact lenses were fitted to younger children compared to non-MC lenses (MC, median 13 years vs. non-MC, median 15 years) $(p<0.0001)$. There was no sex bias in the fitting of MC lenses $(p=0.89)$. Conclusions. MC lenses are currently being prescribed for younger children in equal measure in terms of soft vs. rigid lenses and males vs. females. The extent of MC fitting is low and varies between nations. The gradual increase in MC fitting throughout the survey period perhaps reflects growing concerns among practitioners over the myopia epidemic.
\end{abstract}

Keywords

Taxonomy

Corresponding Author

Corresponding Author's Institution

Order of Authors contact lenses; fitting; myopia control; survey

Contact Lens Prescribing, Soft Contact Lens, Rigid Gas Permeable Lens

Nathan Efron

Queensland University of Texhnology

Nathan Efron, Philip Morgan, Craig Woods, Jacinto Santodomingo-Rubido, Jason Nichols

\section{Submission Files Included in this PDF}

File Name [File Type]

Response to Reviewers.docx [Response to Reviewers (without Author Details)]

Highlights.doc [Highlights]

Title Page.docx [Title Page (with Author Details)]

Manuscript.docx [Manuscript (without Author Details)]

Figure 1.pdf [Figure]

Figure 2.pdf [Figure]

Figure 3.pdf [Figure]

Figure 4.pdf [Figure]

To view all the submission files, including those not included in the PDF, click on the manuscript title on your EVISE Homepage, then click 'Download zip file'.

\section{Research Data Related to this Submission}

There are no linked research data sets for this submission. The following reason is given:

Data will be made available on request 


\title{
Response to Reviewers of the Paper \\ International survey of contact lens fitting for myopia control in children
}

\author{
Reviewers comments in bold font. \\ Our response in plain (non-bold) font \\ Changes in the manuscript highlighted in yellow.
}

Comments from the editors and reviewers:

\section{Guest Editor}

This is an interesting survey to evaluate the extent of prescribing myopia control contact lenses over a 8 year period throughout the world.

Agreed!

Given the current interest in MC by ECPs it is surprising to see the low number of fits for MC in children.

Although the numbers are low, we point out that the rate of $\mathrm{MC}$ prescribing is increasing over time.

Some minor comments from the reviewers need to be considered.

We have considered all comments and responded as indicated below.

In addition how did you determine that practitioners were randomly selected to take part in the survey? - I see details for this for the UK but not for other countries.

This information has been presented in considerable detail in the second and third paragraphs of section 2.1, with various examples of the approach adopted in different countries, aside from the UK

What would be the normal variation in response if the survey was repeated in one year with a different cohort of practitioners? i.e. how does practitioner selection potentially alter the results?

It appears from our experience over 25 years, in conducting this survey in over 60 countries, that year-on-year variances have little impact on the results. Clear, consistent and intuitively plausible trends are observed in respect of numerous parameters assessed, even though, as the Guest Editor correctly surmises, different sets of practitioners are completing the survey each year. Thus, we believe 'practitioner selection' introduces only minimal noise to the overall strong signals observed. 
In the introduction you state the use of surgery, optical or pharmacological interventions for myopia control. Some researchers advocate change in behaviour and that could also be mentioned.

Yes, good point. We have now mentioned this (outdoor light exposure) and introduced a new reference (reference 6) in support.

The timeline for introduction of MiSight CLs to the market was much earlier in Spain and Australia compared to the UK for example and this may reflect some of your findings.

Another good point, although it is even more complicated than this. MiSight was not available in all countries surveyed, and was introduced into some countries at different stages throughout the 8-year course of the work. At the time of writing this paper, the MiSight lens was available in only 14 of the 31 countries surveyed. We have now mentioned this in the paper (first paragraph of Discussion), and have highlighted in the caption to Figure 1 those countries where MiSight was available at the time of writing. This information will assist readers in relating our data to the availability of MiSight.

Some countries have no access to atropine for MC even if it is off-label i.e. UK.

This is true; however, we have deliberately avoided detailed discussion of surgical, pharmaceutical and behavioural approaches to myopia control in this paper, which is of course about contact lens prescribing for myopia control. Detailed discussions of these other approaches are beyond the scope of this paper, apart from the very brief mention in the Introduction to provide broad context.

\section{$\underline{\text { Reviewer } 1}$}

This manuscript describes a survey that was administered over an 8-year period to determine the extent of contact lenses fitted for myopia control around the world. I have some reservations about the methodology - there is little justification for the techniques used to analyse the date. I have some other comments below:

We have responded below to the details raised.

Line 44: using atropine and pirenzepine is also considered off-label use for myopia control

This is true, and we have added this point to the sentence in question.

Line 45: remove peripheral. This is one of the mechanisms by which optical strategies is believed to induce myopia control effects

We have now qualified this statement by noting that this is one possible mechanism of myopia control. We have also replaced the reference of Charman and Radhakrishnan with a more appropriate reference that highlights this debate (the details of which are beyond the scope of this paper). 
Line 96: how did authors determine the threshold value of 100 fits?

There is no 'hard and fast' rule to determine this threshold but this was an attempt to rule out markets with small responses to eliminate spurious inter-country comparisons. Our data allows for the estimate of myopia control lens fitting to have $95 \%$ confidence limits of $+/-$ $10 \%$ of fits or better (lower) which, in our judgement, allows for meaningful comparisons between markets.

\section{Line 100: is calculation of this weighting factor a verified technique?}

The weighting of lens fits is an effort to provide better estimates of prescribing trends. Clearly, a practitioner who completes 10 lens fits in one day is very likely to be prescribing more contact lenses in a year than someone who takes one month to reach the same number of fits. We believe that our approach appropriately caters for this situation. It is not clear how this might be formally 'validated' so instead, we clearly report our method for readers to take into account when they consider the paper. We are also reassured by the observation that this same approach - carefully outlined each time - has featured in approximately 50 refereed scientific papers and 70 clinical articles which we have written in this area over the past 25 years.

Line 123: I find it unusual that places such as Japan and South Korea, which have significantly high myopia prevalence rates, recorded no contact lens fits for myopia control?

We speculate this could be due to the fact that myopia control on the hole at the moment requires off label prescribing and these two countries are not known for their poor compliance to regulatory control.

Discussion: there should be some discussion about the limitations/assumptions made in methodology.

We have introduced a discussion of the limitations of this work in the last paragraph of the Discussion section of the paper.

\section{Reviewer 2}

This is a relatively straightforward article that uses a subset of data, collected as part of a large international survey, to examine the extent of myopia control management that occurs with contact lenses in different countries. The results are to some extent interesting and highlight the different approaches of countries to myopia control. In addition the results may reflect the extent to which contact lens companies such as Coopervision have gained access to specific countries. I have a few comments that the authors should sonsider prior to publication.

We have addressed these comments, as indicated below. 


\section{Introduction}

Line 44: use MC instead of myopia control

We have replaced 'myopia control' with MC.

Line 45: The sentence relating to peripheral positive spherical aberration is too simplistic and is not a true representation of reference 9.

Fair comment. We have re-edited this sentence and introduced a new reference to reflect the ongoing discussion about the aetiology of myopia control. See our response to the Guest Editor who raises a similar point.

\section{Methods}

Line 78: I think the authors should just say that the survey was conducted in the first few months of each year. The text relating to seasonal influences etc implies a degree of control which is not apparent when you consider the differences in climates, seasons etc in the countries surveyed.

We have made this change.

\section{Results}

Line 129: The "overall extent" is confusing - the proportion that MC fits has increased would be more informative.

We have made this change.

\section{Discussion}

Line 192 and 202: the term "myopia epidemic" is mentioned initially in relation to East and South East Asia (ref 10) and then less specifically (ref 16). I think the authors should be careful when discussing this as line 202 gives the impression that the epidemic is global.

Yes, we have re-worded the text and referred to the "growing awareness among eye care practitioners of the importance of arresting myopia progression where possible".

Line 176 - this statement needs to be discussed. Were the soft lens MC values higher in countries where MiSight was introduced earlier? Relate this to examples from figure 1 to provide more evidence.

This is a good point. Please see our response to the Guest Editor, who essentially raised the same issue. 
Figure 1 The large error bars in figure 1 for AT, DE and HK suggest that there has been a large change in MC prescribing over the survey period

These large error bars reflect great year-to year variance in MC prescribing in these countries. However, this variance has not precluded the demonstration of highly significant differences between countries, and we have presented the statistical evidence for this.

Figure 3 legend: "as a proportion of all soft and rigid lens fits in children". It would also be useful to have an idea of the $n$ number here as percentages can be confusing.

We feel that this graph is abundantly clear. 'Percentages' are a well-understood statistical descriptor. To include the 16 ' $n$ ' data values in the graph and/or caption would be cumbersome, would make this figure unwieldy, and would seriously detract from the message we are trying to deliver. The total ' $n$ ' for this work is given in the Results section.

\section{The data for 2013 is also worthy of mention.}

Data for 2013 is the same as data for 2011. By the same token, data for 2012 is the same as that for 2014 and 2015. The reviewer seems to be suggesting the 2012 data is spurious. But the same could have been said about the 2013 data. The point here is that there will always be variances in data, that cannot always be easily explained; it is important to focus on overall trends, which we have done.

Figure 4 legend: this legend requires more detail. Again, an n number would be useful for the two groups when dealing with proportions.

It is the proportion of wearers that is important here, which serves as a valuable tool for normalising the graph for ready comparison of $\mathrm{MC}$ and non-MC age data. To include the 34 ' $n$ ' data values in the graph and/or caption would be cumbersome, would make the graph unwieldy, and would seriously detract from the message we are trying to deliver. The total ' $n$ ' for this work is given in the Results section. 


\section{Highlights}

- A survey of contact lens prescribing for myopia control in children was conducted annually in 31 countries between 20011 and 2018, inclusive.

- Myopia control fits represented 2.3\% of all contact lens fits to children, although the extent of myopia control fitting has slowly increased over time.

- A similar proportion of females (58.1\%) ws fitted with myopia control lenses versus nonmyopia control lenses $(62.3 \%)$.

- The median age of myopia control and non- myopia control fits is 13 and 15 years, respectively, with most myopia control fitting activity taking place among children aged 8 to 15 years.

- Of all contact lenses fitted for the purpose of myopia control to children, $52.1 \%$ were undertaken using rigid lenses; this compares with $12.0 \%$ for non- myopia control fits with rigid lenses. 


\section{International survey of contact lens fitting for myopia control in children}

Nathan Efron ${ }^{\mathrm{a}}$, Philip B. Morgan ${ }^{\mathrm{b}}$, Craig A. Woods ${ }^{\mathrm{c}}$, Jacinto Santodomingo-Rubido ${ }^{\mathrm{d}}$, Jason J.

Nichols ${ }^{\mathrm{e}}$ and The International Contact Lens Prescribing Survey Consortium

anstitute of Health and Biomedical Innovation, and School of Optometry and Vision Science,

Queensland University of Technology, Australia

${ }^{b}$ Eurolens Research, Division of Pharmacy and Optometry, The University of Manchester, UK

${ }^{\mathrm{c} S c h o o l}$ of Medicine (Optometry), Deakin University, Australia

dMenicon Co., Ltd., Nagoya, Japan

'The University of Alabama at Birmingham, School of Optometry, Birmingham, Alabama, USA

*Corresponding author at:

Institute of Health and Biomedical Innovation, and School of Optometry, Queensland University of Technology, Kelvin Grove, Queensland 4059, Australia.

E-mail address: n.efron@qut.edu.au (N. Efron)

4 Figures, 1 Table

Running header: Contact lens myopia control survey 
2 International survey of contact lens fitting for myopia control in children 3 


\section{ABSTRACT}

Purpose. To determine the extent of contact lens fitting for myopia control (MC) in children (defined as $\leq 17$ years of age) worldwide and to characterize the associated demographics and fitting patterns.

Methods. Survey forms were sent to contact lens fitters in 66 countries between January and March every year for eight consecutive years (2011 to 2018, inclusive). Practitioners were asked to record data relating to the first 10 contact lens fits performed after receiving the survey form. Data were analysed for those countries reporting $\geq 100$ contact lens fits to children. Results. Data were analysed for $535 \mathrm{MC}$ fits and 23,830 other (non-MC) lens fits undertaken in 31 countries reporting $\geq 100$ contact lens fits to children, with $52.1 \%$ of $\mathrm{MC}$ fits and $12.0 \%$ of non-MC fits being with rigid lenses $(\mathrm{p}<0.0001)$. Overall, MC lenses represented $2.3 \%$ of all contact lens fits to children, with significant differences between nations $(\mathrm{p}<0.0001)$, ranging from no MC fits recorded in the Czech Republic, Greece, Japan, South Korea and Puerto Rico, to 24.9\% in Austria. There has been an increase in contact lens fitting for MC over the survey period $(\mathrm{p}<0.0001)$. MC contact lenses were fitted to younger children compared to non-MC lenses (MC, median 13 years vs. non-MC, median 15 years $)(p<0.0001)$. There was no sex bias in the fitting of MC lenses $(\mathrm{p}=$ $0.89)$.

Conclusions. MC lenses are currently being prescribed for younger children in equal measure in terms of soft vs. rigid lenses and males vs. females. The extent of MC fitting is low and varies between nations. The gradual increase in MC fitting throughout the survey period perhaps reflects growing concerns among practitioners over the myopia epidemic.

Key words: contact lenses, fitting, myopia control, survey. 


\section{Introduction}

The term 'myopia control' (MC) has been coined to describe the practice of introducing an intervention to arrest the progression of myopia. There are four fundamental approaches to MC surgical, pharmacological, behavioural, and optical. Surgical approaches have focused on posterior scleral cross-linking and appear to be applied to high levels of myopia [1-3]. Pharmacological approaches have centered around the use of the anti-muscarinic agents atropine [4] and pirenzepine [5]; these drugs have been demonstrated to partially arrest the progression of myopia in children, although the precise mechanism by which this occurs is unclear. Behavioural change relates to the notion that spending more time outdoors in sunlight can arrest the rate of progression of myopia [6].

Optical approaches include the use of bifocal or multifocal spectacles [7], and concentric ring bifocal or peripheral-add multifocal soft contact lenses that incorporate additional plus power in the lens periphery [8]. Orthokeratology contact lenses have also been reported as being able to arrest myopia progression, in addition to the classical myopia correction effect of this modality [9]. It should be noted that in most parts of the world, MC using spectacles, contact lenses and pharmaceutical agents (atropine and pirenzeprine) is considered an 'off-label' (unregulated) use of a medical device or drug. Positive spherical aberration induced by these optical configurations is one possible mechanism that is thought to create a reduction in the stimulus for eye growth [10].

The increasing prevalence of myopia around the world - especially in Asian regions [11] - and the awareness of ocular pathology associated with high myopia [12], have heightened interest in clinical management strategies to arrest myopia progression. This paper examines trends in, and factors associated with, contact lens fitting for MC in children ( $\leq 17$ years of age [13]) in 31 countries over an eight year period (2011 to 2018, inclusive). Such information can provide (a) a valuable yardstick for contact lens clinicians, against which they can assess their own prescribing approaches to MC, and (b) useful guidance to the contact lens industry on the clinical utilization of this relatively new product category.

\section{Materials and methods}

\subsection{Conduct of the annual survey}

Between January and March each year from 2011 to 2018, a contact lens fitting survey was undertaken in 66 countries. This was achieved through the offices of members of the International Contact Lens Prescribing Survey Consortium, which is a network of academics, industry representatives, and clinical colleagues who have agreed to manage the survey in their country or geographic region, as outlined below. 
Each Consortium member was requested to send a paper or electronic (e-mail) survey form to as many contact lens practitioners (opticians, optometrists and/or ophthalmologists, depending on the market) in their country as possible, with a notional target of 1,000 surveys sent. Some smaller countries were unable to reach this target due to the limited number of practitioners in that region.

In some countries, such as Australia, survey forms were sent by e-mail to virtually every practitioner in the country (about 5,000). In other countries, survey forms were sent to a selection of practitioners throughout the country; this process varied among countries, ranging for example from a computer-generated random list of 1,000 recipients from the national practitioner register in the UK, to a list of practitioner-subscribers to contact lens trade publications in the USA and Russia. In larger countries with dispersed populations, such as China and Russia, survey forms were sent to practitioners in a select number of major urban cities. The survey forms were sent together with a request that they be completed and returned within three months of receipt. The survey was conducted in the first few months of each year in all countries.

A standardized, one-sided, locally-translated survey form was used each year. Practitioners were requested to enter a number of background details and to supply generic information about the first ten contact lens fits performed after receipt of the survey form (Table 1). Practitioners were asked to return the form irrespective of the number of patients seen (if fewer than 10). The returned forms were logged and data from each form were manually entered into an Excel spreadsheet (Microsoft Corporation, Redmond WA) to facilitate data analysis.

The Office of Research Ethics at the University of Waterloo, Canada, granted approval for this work, and noted that, as the data being collected were (1) part of normal practice care provision, (2) transcribed from the practice patient records, and (3) de-identified in respect of the patients and source practice, then the requirement for patient informed consent was waived

\subsection{Statistical analysis}

Data were analysed for all contact lens fits to children, defined as those $\leq 17$ years of age [13], in countries where $\geq 100$ fits were reported. Differences among practitioners in relation to the amount of contact lens fitting performed were accounted for by assigning an appropriate weighting to each recorded contact lens fit. This was achieved by estimating the number of contact lens fits performed each year by each respondent (based on the date information provided on the survey form) and using this as a weighting factor. For example, the data generated by a practitioner completing all 10 
fits in one week were given twice the weighting of a practitioner who fitted 10 patients in two weeks. Data were mined with the aid of the Excel $^{\circledR}$ Pivot Table function.

Analysis of the main differences in the dataset were conducted using logistic regression models (JMP, SAS Institute Inc., Cary NC, USA) to compare differences between children fitted with MC lenses versus those fitted with non-MC lenses. Further to this, a sub-analysis was conducted within the $\mathrm{MC}$ group only to compare differences in age and sex for those prescribed rigid lenses compared with those prescribed soft lenses. These differences were assessed statistically using a Mann Whitney U test and a chi-square test, respectively.

\section{Results}

\subsection{Data collection}

Over the 8 year survey period (2011 to 2018, inclusive), 31 eligible countries provided data for at least 100 contact lens fits to children, resulting in a total of 23,830 fits. In this sample, there were 535 contact lens fits designated as being for the purpose of MC and 22,295 standard lens fits (i.e. 'non-MC'; these data were used in this study for comparison purposes). The median country response rate was 769 fits, ranging from 115 fits in Puerto Rico to 6,994 fits in Japan.

\subsection{Participating countries}

Averaged over the eight year survey period, contact lens fits for MC represented $2.3 \%$ of all contact lens fits to children; however, there was considerable variance in MC lens fitting among the 31 eligible countries surveyed $\left(\chi^{2}=539, \mathrm{p}<0.0001\right)$, ranging from no fits recorded in the Czech Republic, Greece, Japan, South Korea and Puerto Rico, to 24.9\% in Austria (Fig. 1). It can be seen from Figure 1 that, of the four countries with the highest rate of MC fitting to children, three were Germanic countries - namely, Austria, Germany and Switzerland. This is also evident in Fig. 2, which is a color-coded 'heat map', showing the extent of MC fitting around the world.

\subsection{Trends over time}

The proportion of MC contact lens fitting to children has risen through the eight year survey period $\left(\chi^{2}=203, p<0.0001\right)$, increasing from $0.2 \%$ of all lens fits in 2011 to $6.8 \%$ in 2018 .

Figure 3 shows the change in soft and rigid MC fits to children as a proportion of all soft and rigid lens fits, respectively. Evident in this figure is the sharp increase, especially over the last three years of this survey (2016 to 2018), in extent of rigid lens fits for MC as a proportion of all rigid lens fits. 


\subsection{Patient age}

The distribution of MC and non-MC lens fits, stratified by age, is presented in Fig. 4. It is evident from inspection of this plot that the distribution of MC fits is skewed towards a younger age than the distribution of non-MC fits; this difference is statistically significant $\left(\chi^{2}=69, \mathrm{p}<0.0001\right)$. The median age of MC and non-MC fits is 13 and 15 years, respectively. Most MC fitting activity appears to be taking place among children aged 8 to 15 years.

\section{$3.5 \mathrm{Sex}$}

There was no significant difference in the proportion of females fitted with MC lenses $(58.1 \%)$ versus those fitted with non-MC lenses $(62.3 \%)\left(\chi^{2}=0.02, p=0.89\right)$.

\subsection{Lens type}

The conduct of this survey precluded a precise determination of the lens designs used for MC; however, it is assumed that essentially all rigid lenses fitted for MC to children were orthokeratology designs and all soft lenses fitted for MC were centre-distance multifocal designs.

Of all contact lenses fitted for the purpose of MC to children, 52.1\% were undertaken using rigid lenses; this compares with $12.0 \%$ for non-MC fits with rigid lenses $\left(\chi^{2}=143, \mathrm{p}<0.0001\right)$.

\subsection{Frequency of lens wear}

The frequency of lens wear was $6.7 \pm 0.9$ days per week for MC fits versus $5.8 \pm 1.7$ days per week for non-MC fits $\left(\chi^{2}=116, \mathrm{p}<0.0001\right)$. Lenses were worn 7 days per week by $84 \%$ of children fitted with MC lenses versus $56 \%$ of children fitted with non-MC lenses.

\subsection{Differences within MC group}

Differences found between children for the two main types of MC lenses fitted were not statistically significant. Median age was similar for the two lens types (12 years for children using rigid lenses for $\mathrm{MC}$ versus 13 years for children wearing soft lenses $)(Z=1.8, \mathrm{p}=0.08)$. The difference in breakdown for sex (54\% female in those wearing rigid lenses and $60 \%$ female among soft lens wearers) was also not significant $\left(\chi^{2}=2.1, \mathrm{p}=0.14\right)$.

\section{Discussion}

International differences in the extent of contact lens fitting for MC may be attributed to a number of factors. Throughout the period of this survey, the MiSight lens (CooperVision, USA) [14] was the only soft lens specifically approved for the indication of MC. This lens was not available in all 
countries surveyed, but was introduced into some countries at different stages throughout the 8-year course of the work; at the time of writing, the MiSight lens was available in only 14 of the 31 countries surveyed (see Figure 1). Nevertheless, some practitioners appear to have elected to prescribe 'off label' lens designs licensed to correct presbyopia, for the purposes of MC in children; the present data confirm this, as a number of countries where MiSight was not available at any stage during the survey period reported soft lens use for MC in children. Regardless, increased fitting for MC would be expected to be observed in those countries in which the MiSight lens was available, and moreso where the MiSight lens was introduced earlier.

International differences in the training, attitudes and collective confidence of the predominant eye care practitioner groups - opticians, optometrists and ophthalmologists - and the proportion of practitioner type in different regions, may also impact the extent of contact lens MC fitting between countries. For example, there may be a higher rate of prescribing of low dose atropine, and a commensurate lower prescribing rate of contact lenses, in regions with a high proportion of ophthalmologists or therapeutically-endorsed optometrists.

An unexpected finding of this survey was the relatively high rate of $\mathrm{MC}$ prescribing for children in the Germanic countries of Austria, Germany and Switzerland. This may be attributed to two factors: (1) the traditional high prescribing rate of rigid lenses in central Europe and the commensurate possession of advanced rigid lens fitting skills set among European practitioners (compared to Asia), who are capable of fitting orthokeratology rigid lenses for $\mathrm{MC}$ and have ready access to specialised local rigid lens laboratories who can supply such lenses, coupled with (2) the availability of the MiSight lens for MC in these three countries. Highest rates of MC prescribing might have been predicted to be observed in parts of east and southeast Asia in view of the welldocumented myopia epidemic in this region [11]. Perhaps difficulties in obtaining regulatory approval in some Asian markets (e.g. Japan) have served to constrain MC fitting in this region.

Overall, MC represented only $2.3 \%$ of all contact lens fits, notwithstanding the gradual increase in MC fitting throughout the survey period. Three factors that may have limited the uptake of contact lenses for MC are (1) the lack of on-label MC products for most of the survey period and in most parts of the world; (2) reservations and uncertainty as to the true efficacy of MC fitting [15], and (3) an apparent lack of awareness among parents of myopic children of the ocular health risks of myopia development and the potential benefits of MC [16]. Nevertheless, the gradual increase in MC fitting over the course of this survey possibly reflects a growing awareness among eye care practitioners of the importance of arresting myopia progression where possible [17]. 
The skewed age distribution of MC fits towards younger children as observed in this study is consistent with the notion that $\mathrm{MC}$ lenses should be prescribed at the earliest possible sign of myopia development [18].

Despite the higher prevalence of myopia among Caucasian and East Asian adolescent females [19], no difference was observed in the proportion of females fitted with MC versus non-MC lenses in the present study. The reason for the apparent under-representation of females is unclear; one possible explanation is that a higher proportion of females is being treated using alternative MC strategies, such as low-dose atropine.

Although rigid lenses specifically approved for MC were not available during the survey period, the high rate of rigid lens fitting for MC (compared with non-MC rigid lens fits) - which are essentially orthokeratology lens fits - may be due to a number of factors: (1) the possession of high levels of orthokeratology lens fitting skills in some regions, as discussed above; (2) the ready availability of local laboratories able and willing to supply appropriate orthokeratology designs for MC fitting; and (3) the belief among some practitioners that orthokeratology is more efficacious than soft lens fitting for MC.

That MC lenses are worn about one day per week more than non-MC lenses is consistent with the higher rate of rigid (orthokeratology) lens fits for $\mathrm{MC}$ versus non-MC. Orthokeratology lenses must be worn every night to maintain the daily corneal moulding effect required to improve vision [20]. Also, rigid lenses are not conducive to intermittent lens wear; it is well accepted that full-time lens wear helps maintaining lens adaptation, thus enhancing day to day comfort [21]. The higher frequency of lens wear for MC compared to that for non-MC may also relate to a perception, in the absence of any direct evidence, that MC is dose-dependent, i.e. the more such lenses are worn, the greater is the efficacy of the procedure for arresting myopia progression.

No clear differences were seen the demographics of children fitted with soft lenses versus rigid lenses for MC. This is unsurprising for the breakdown in sex between the two lens types, but it is also informative that there is no clear trend at this time for practitioners to select a soft lens in younger MC patients on the basis that this might be a simpler or more familiar option for a potentially challenging patient population. 
Sampling bias is perhas the greatest limitation of this study. In many contries investigated, survey distribution is not completely random; for example, in many instances, surveys are sent to known groups of practitioners with a special interest in contact lenses (as distinct from 'general' eye care practitioners). Therefore, this could result in a bias towards the survey data reflecting the prescribing data of those with a particular interest in contact lens prescribing. Also, it is assumed that survey respondents have reported accurately; the nature of the study is such that it has not been possible to audit the submitted data. Despite these limitations, the clear, consistent and intuitively plausible trends observed in respect of numerous parameters assessed suggests that the data set is robust.

\section{Conclusions}

Contact lens fitting to arrest the progression of myopia in children is one of a number of viable strategies available for fighting the myopia epidemic. This approach has been adopted by eye care practitioners in many nations over the past eight years at a relatively low level, although there is evidence of a gradual increased MC fitting over time. Rigid lenses (primarily orthokeratology) and soft lenses (primarily centre-distance multifocals) are being fitted for $\mathrm{MC}$ to children in approximately equal measure. Children being fitted with $\mathrm{MC}$ lenses are younger than those being fitted with non-MC lenses.

\section{Acknowledgments}

The International Contact Lens Prescribing Survey Consortium: Nathan Efron, Australia; Philip B Morgan, United Kingdom; Craig A Woods, Australia; Jacinto Santodomingo-Rubido, Spain; Jason J. Nichols, United States of America; Carmen Abesamis-Dichoso, The Philippines; Waleed Aighamdi, Saudi Arabia; Suresh Awasthi, Nepal; Joseph Barr, United States of America; Marion Beeler-Kaupke, Switzerland; Jitka Belikova, The Czech Republic; Vadim Belousov, Russia; Jolanta Bendoriene, Lithuania; Janet Casablanca, Puerto Rico; Aris Chandrinos, Greece; Prema Chande, India; Patrick Cheng, Hong Kong; JhoYan Chia, Malaysia; Byoung Sun Chu, South Korea; Edgar Dávila-Garcia, Puerto Rico; Nir Erdinest, Israel; Chi Shing Fan, Asia-Pacific; Philip Fine, Israel; Peter Gierow, Sweden; José Manuel González-Méijome, Portugal; Martha Yanneth Gonzalez, United States of America; Hans-Jürgen Grein, Germany; Christina N Grupcheva, Bulgaria; Jörgen Gustafsson, Sweden; Magne Helland, Norway; Anna Yeo Chwee Hong, Singapore; Hreinn Ingi Hreinsson, Iceland; John Hsiao, Taiwan; Lee Kai Hung, Singapore; Motozumi Itoi, Japan; Ali Reza Jafari, Iran; Oskar Johansson, Sweden; Deborah Jones, Canada; Lyndon Jones, Canada; Razmig Knajian, United Arab Emirates; Jitka Krasnanska, The Czech Republic; Wanda Lam, New Zealand;

Weizhong Lan, China; Rafael S. Lemos, Brazil; Weronika Leszczyńska, Poland; Carla J Mack, 
United States of America; Patricia Magnelli, Argentina; Florence Malet, France; Edoardo Marani, Italy; Sebastian Marx, Germany; Nelson L. Merchan, Colombia; Giancarlo Montani, Italy; Jose L. Moroy; Mexico; Vishakh Nair, Saudi Arabia; Alex Ong, Singapore; Guillermo Carrillo Orihuela, The Americas; Alice Pesinova, The Czech Republic; Anna Lindskoog Pettersson, Sweden; Geraint Phillips, New Zealand; Ricardo Pintor, Mexico; Athina Plakitsi, Greece; Heiko Pult, Germany; Polo Qi, China; Simona Radu, Romania; Jona Birna Ragnarsdóttir, Iceland; Hrvoje Raguž, Croatia; Raul

L Ramos Gómez; Ole Ravn, Denmark; Jeanette Romualdez-Oo, The Philippines; Svend-Erik Runberg, Denmark; Mirna Stabuc Silih, Slovenia; Danny Sim, Singapore; Leon Sze, Hong Kong; Kah-Ooi Tan, China; Panu Tast, Finland; Mario Teufl, Austria; Inga-Lill Thunholm-Henriksson, Sweden; Ioannis G Tranoudis, Greece; Marco van Beusekom, The Netherlands; Eef van der Worp, The Netherlands; Mihály Végh, Hungary; Edit Vodnyanszky, Hungary; Ann Elisabeth Ystenæs, Norway; and Veronica Ziziuchin, Moldova.

\section{References}

[1] B. Ward, E.P. Tarutta, M.J. Mayer, The efficacy and safety of posterior pole buckles in the control of progressive high myopia. Eye 23 (12) (2009) 2169-2174.

[2] A. Elsheikh, J.R. Phillips, Is scleral cross-linking a feasible treatment for myopia control? Ophthal Physiol Optics 33 (3) (2013) 385-389.

[3] A. Xue, L. Zheng, G. Tan, S. Wu, Y. Wu, L. Cheng, J. Qu, Genipin-crosslinked donor sclera for posterior scleral contraction/reinforcement to fight progressive myopia. Invest Ophthalmol Vis Sci 59 (8) (2018) 3564-3573.

[4] W.H. Chua, V. Balakrishnan, Y.H. Chan, L. Tong, Y. Ling, B.L. Quah, D. Tan, Atropine for the treatment of childhood myopia. Ophthalmology 113(12) (2006) 2285-2291.

[5] D.T. Tan, D.S. Lam, W.H. Chua, D.F. Shu-Ping, R.S. Crockett, Asian Pirenzepine Study Group, One-year multicenter, double-masked, placebo-controlled, parallel safety and efficacy study of 2 per cent pirenzepine ophthalmic gel in children with myopia. Ophthalmology 112 (1) (2005): 84-91.

[6] P.C. Wu, C.T. Chen, K.K. Lin, C.C. Sun, C.N. Kuo, H.M. Huang, Y.C. Poon, M.L. Yang, C.Y. Chen, J.C. Huang, P.C. Wu, I.H. Yang, H.J. Yu, P.C. Fang, C.L. Tsai, S.T. Chiou, Y.H. Yang, Myopia prevention and outdoor light intensity in a school-based cluster randomized trial. Ophthalmology. 125 (8) (2018) 1239-1250.

[7] P. Sankaridurg, L. Donovan, S. Varnas S,A. Ho, X. Chen, A. Martinez, S. Fisher, Z. Lin, E.L. Smith $3^{\text {rd }}$, J. Ge, B. Holden, Spectacle lenses designed to reduce progression of myopia: 12-month results. Optom Vis Sci 87 (9) (2010) 631-641. 
S.M. Li, M.T. Kang, S.S. Wu, B. Meng, Y.Y. Sun, S.F. Wei, L. Liu, X. Peng, Z. Chen, F. Zhang, N. Wang, Studies using concentric ring bifocal and peripheral add multifocal contact lenses to slow myopia progression in school-aged children: a meta-analysis. Ophthalmic Physiol Opt 37 (1) (2017) 51-59.

9] H.A. Swarbrick, A. Alharbi, K. Watt, E. Lum, P. Kang, Myopia control during orthokeratology lens wear in children using a novel study design. Ophthalmology 122 (3) (2015) 620-630.

[10] E.L. Smith 3rd, M.C. Campbell, E. Irving, Does peripheral retinal input explain the promising myopia control effects of corneal reshaping therapy (CRT or ortho-K) \& multifocal soft contact lenses? Ophthalmic Physiol Opt 33 (3) (2013) 379-84.

[11] B.A. Holden, FT.R. Fricke, D.A. Wilson, M. Jong, K.S. Naidoo, P. Sankaridurg, T.Y. Wong, T.J. Naduvilath, S. Resnikoff, Global prevalence of myopia and high myopia and temporal trends from 2000 through 2050.,Ophthalmology 123 (5) (2016) 1036-1042.

[12] S.M. Saw, G. Gazzard, E.C. Shih-Yen, W.H. Chua, Myopia and associated pathological complications. Ophthalmic Physiol Opt 25 (5) (2005) 381-391.

[13] N. Efron N, P.B. Morgan, C.A. Woods, The International Contact Lens Prescribing Survey Consortium, Survey of contact lens prescribing to infants, children and teenagers. Optom Vis Sci 88 (4) (2011) 461-468.

[14] A. Ruiz-Pomeda, B. Pérez-Sánchez, I. Vallas, F.L. Prieto-Garrido, R. Gutiérrez-Ortega, C. Villa-Collar, MiSight assessment study spain (MASS). A 2-year randomized clinical trial. Graefes Arch Clin Exp Ophthalmol 256 (5) (2018) 1011-1021.

[15] N.A. Brennan, X. Cheng, Commonly held beliefs about myopia that lack a robust evidence base. Eye Contact Lens (in press) doi: 10.1097/ICL.0000000000000566

[16] S. McCrann, I. Flitcroft, K. Lalor, J. Butler, A. Bush, J. Loughman, Parental attitudes to myopia: a key agent of change for MC? Ophthalmic Physiol Opt 38 (3) (2018) 298-308.

[17] J.S. Wolffsohn, A. Calossi, P. Cho, K. Gifford, L. Jones, M. Li, C. Lipener, N.S. Logan, F. Malet, S. Matos, J.M. Meijome, J.J. Nichols, J.B. Orr, J. Santodomingo-Rubido, T. Schaefer, N. Thite, E. van der Worp, M. Zvirgzdina, Global trends in myopia management attitudes and strategies in clinical practice. Cont Lens Anterior Eye 39 (2) (2016) 106-116.

[18] P. Cho, S.W. Cheung, Protective role of orthokeratology in reducing risk of rapid axial elongation: A reanalysis of data from the ROMIO and TO-SEE studies. Invest Ophthalmol Vis Sci 58 (3) (2017) 1411-1416.

[19] R.A. Rudnicka, V.V. Kapetanakis, A.K. Wathern, N.S. Logan, B. Gilmartin, P.H. Whincup, D.G. Cook, G.G. Owen, Global variations and time trends in the prevalence of 
childhood myopia, a systematic review and quantitative meta-analysis: implications for aetiology and early prevention. Br J Ophthalmol 100 (7) (2016) 882-890. P. Gifford, Orthokeratology. In: Efron N, ed. Contact Lens Practice. 3rd Edition. Edinburgh: Elsevier; 2018: 296-304.

G. Young, Rigid lens design and fitting. In: Efron N, ed. Contact Lens Practice. 3rd Edition. Edinburgh: Elsevier; 2018: 156-162. 


\section{Figure Legends}

Fig. 1. Proportion all contact lenses fitted for MC to children in 31 countries between 2011 and 2018. Error bars represent the positive 95\% confidence limit. AT Austria*, AU Australia*; BG Bulgaria; CA Canada*; CH Switzerland*; CZ Czech Republic; DE Germany*; DK Denmark*; ES Spain*; FR France*; GR Greece; HK Hong Kong*; HU Hungary; IL Israel; IT Italy; JP Japan; KR South Korea; LT Lithuania; MX Mexico, NL Netherlands*; NO Norway*; NZ New Zealand; PH Philippines; PR Puerto Rico; PT Portugal*; RU Russia; SE Sweden*; SI Slovenia; TW Taiwan; UK United Kingdom*; US United States. *MiSight lens available at time of writing.

Fig. 2. 'Heat map' indicating the extent of MC lens fitting to children in different countries around the world. Note the logarithmic colour-coded scale. Nations shown in white (no colour) were not surveyed.

Fig. 3. Trend in the proportion of soft and rigid MC fits to children as a proportion of all soft and rigid lens fits, respectively, in 31 countries between 2011 and 2018. Error bars represent the $95 \%$ confidence limit.

Fig. 4. Age categories of children fitted with contact lenses for MC, and for children fitted with all other lens types (non-MC), in 31 countries between 2011 and 2018. 
374 Information captured about each patient fitted.

\begin{tabular}{|l|l|}
\hline Information category & \multicolumn{1}{|c|}{ Options } \\
\hline Date of fitting & (day/month) \\
\hline Age & (in years) \\
\hline Sex & Male \\
& Female \\
\hline Type of fit & New fit \\
& Refit \\
\hline Rigid lens material/type & Scleral \\
& PMMA \\
& Dk $<40$ \\
& Dk 40-90 \\
& Dk $>90$ \\
\hline Soft lens materials & Conventional hydrogel $<40 \%$ water \\
& Conventional hydrogel 40-60\%water \\
& Conventional hydrogel $>60 \%$ water \\
& Silicone hydrogel \\
\hline Lens design & Sphere \\
& Toric \\
& Multifocal \\
& Monovision \\
& Cosmetic tint \\
& Orthokeratology \\
& Myopia control \\
& Other \\
\hline Times per week of wear & Daily \\
\hline Modality & $1-7$ \\
\hline Care system & Daily wear \\
& Extended wear \\
\hline \multirow{5}{*}{ Frequency of replacement } & Multipurpose \\
& Peroxide \\
& Othenth months \\
& None \\
\hline & \\
& Unplanned \\
\hline
\end{tabular}




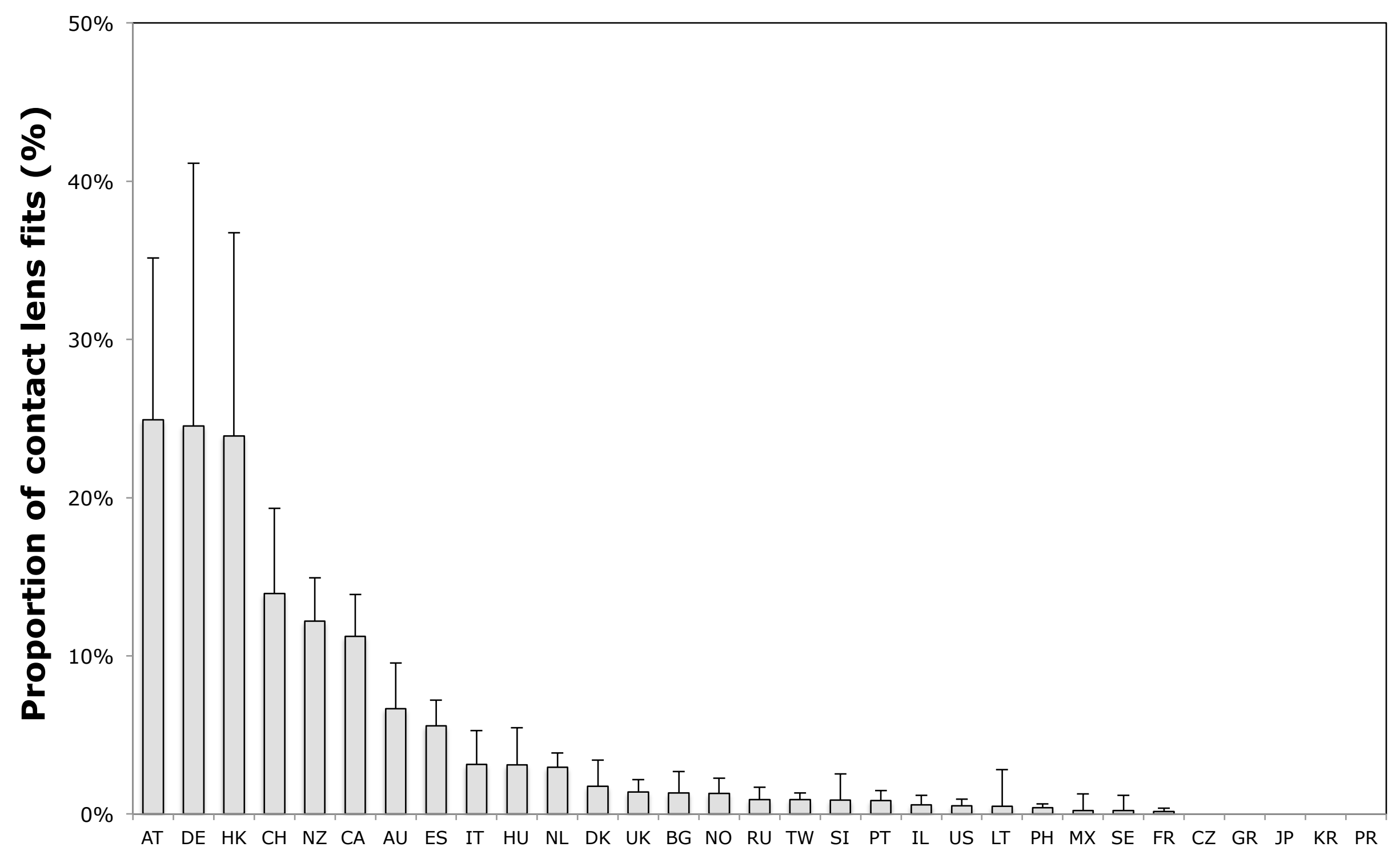




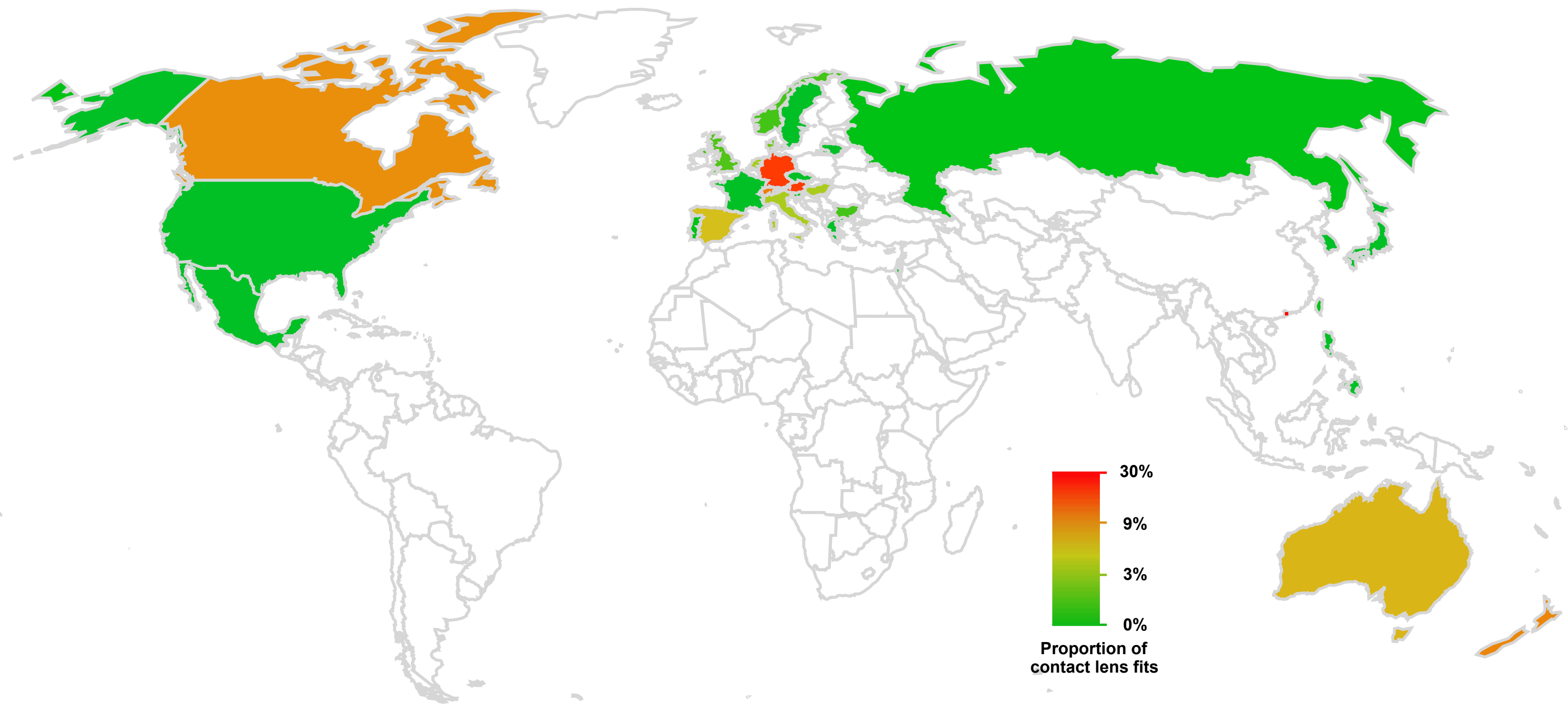




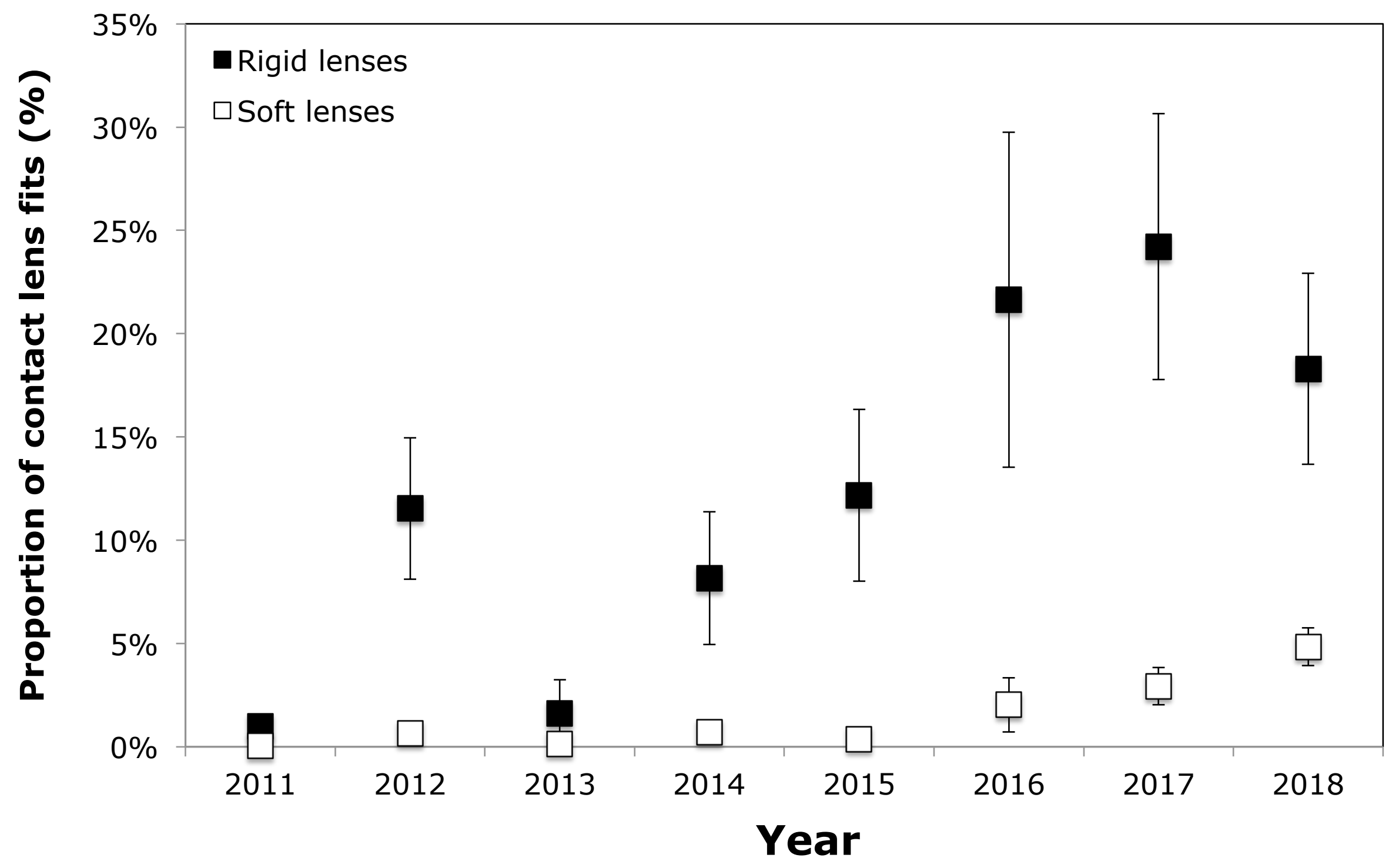




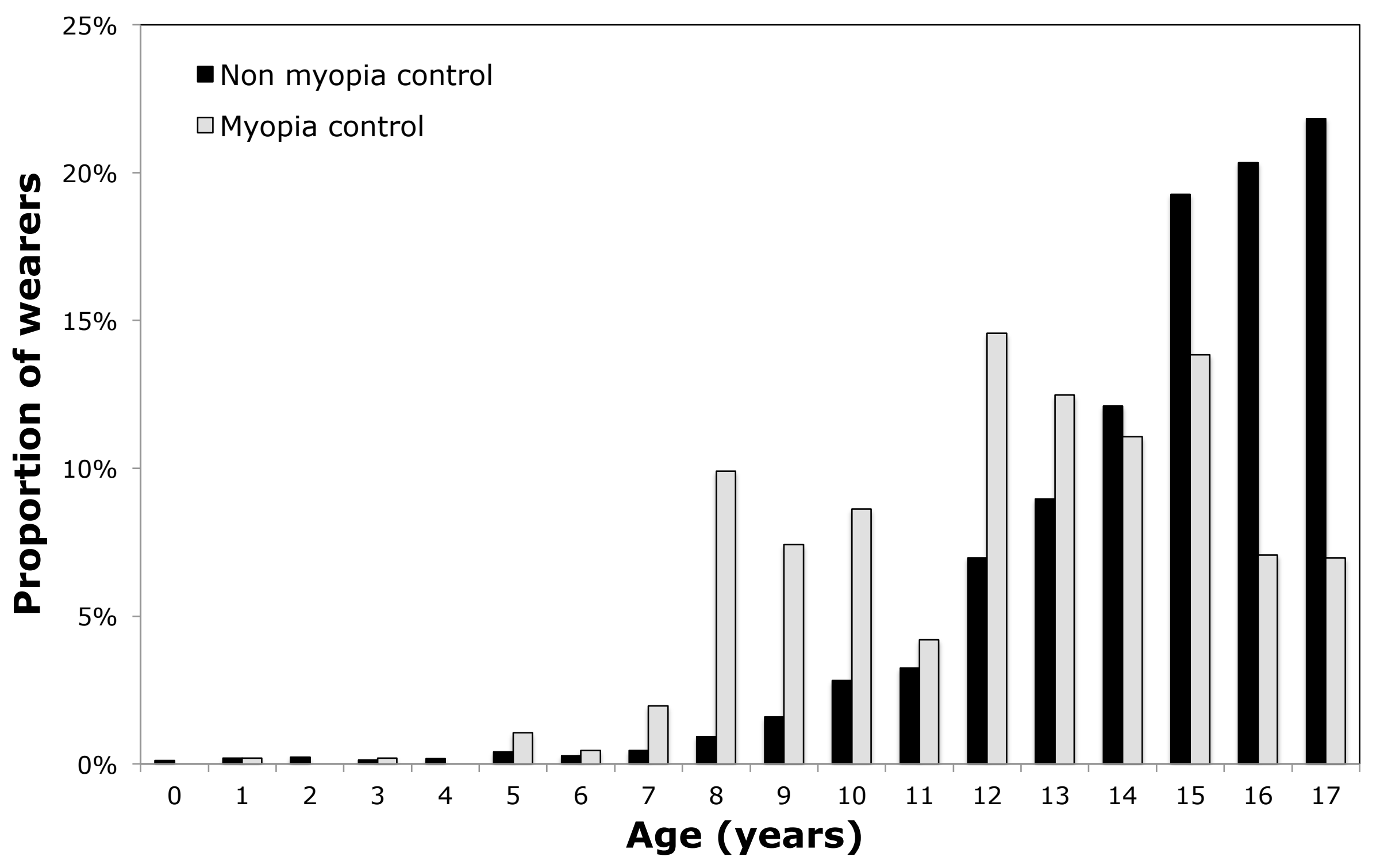

